\title{
Assessment of association of postprandial blood sugar with lipid parameters in non-diabetic central Indian population
}

\author{
Jyoti Agrawal ${ }^{1}$, Neelam Bharihoke ${ }^{2}$ \& Anand Kar $^{1}$ \\ ${ }^{1}$ (School of Life Sciences, Devi Ahilya University, Takshshila Campus, Indore 452017, (M.P.) India). \\ ${ }^{2}$ (Department of Pathology, Bombay Hospital, Indore 452010, (M.P.) India).
}

\begin{abstract}
Diabetic condition may enhance the risk of dyslipidemia and cardiac problems, which generally appears to worsen with age. The correlation analysis was performed of postprandial blood sugar (PPBS) with lipid profile parameters in non-diabetic normal population of Indore, Madhya Pradesh, India. In the study, total 726 normal subjects were taken, who came to Bombay Hospital for their general health check up. The data was analysed by the Pearson's correlation analysis. Results showed a non-significant correlation of PPBS with total cholesterol (TC), low density lipoprotein ( $L D L)$ and $L D L$ to high density lipoprotein (HDL) ratio. While a significant positive correlation was observed between PPBS and triglyceride $(r=0.342, P<0.001), T C$ to HDL ratio $(r=0.193, P<0.001)$; and negative correlation with $H D L(r=-0.147, P<0.001)$. The present data showed a moderate correlation of PPBS with triglyceride, $H D L$, and TC/HDL ratio. It might indicate that normal individuals are at increased risk of hyperglycaemia and cardiac abnormalities. Increasing chances of metabolic abnormalities in the given sample population indicate that there is a strong need for screening of general health status in large population size with its management.
\end{abstract}

Key words: postprandial blood sugar, triglyceride, high density lipoprotein, correlation, dyslipidemia

\section{Introduction}

Prevalence of metabolic disorders including diabetes and cardiovascular problems (CVD) are swiftly rising in the world. In India also it is contributing as a leading cause of death. However, the commonness of disorder varies extensively, but on an average it accounts about $28 \%$ of all deaths in India [1,2]. Recent data also found that in India the highest number of deaths occur due to coronary heart diseases [3]. More often these cardiac problems are associated with hyperglycaemia, which is an endocrine metabolic disorder. The abnormalities in normal metabolism lead to increase the incidences of obesity, atherosclerosis and other metabolic syndromes which aggravate with time [4].

Thus abnormality in one may impair the other. Research data also proved that long term abnormalities in the blood glucose and lipid level may permanently damage to the normal metabolism that result in type 2 diabetes mellitus (DM), atherosclerosis, obesity and CVD [1,5-6]. Earlier findings revealed that epidemiological analysis of blood glucose and lipid profiles of different eastern, western, northern and southern Indian regions have demonstrated incidence of growing irregularities in serum glucose and total cholesterol (TC), high-density lipoprotein (HDL), low density lipoprotein (LDL) and cardiac risk ratio (CRR) as well [1,4].

However, chronic diabetes has already been reported to associate with increasing risk of CVD, micro and macro vascular complications [7-8] but with reference to normal (non-diabetic) individuals the relation of PPBS with different lipid parameters has not been documented till now, principally in case of central Indian population no data existed. Thus, in this study the correlation of PPBS with various lipid parameters was analysed in healthy urban population of Indore, central India.

\section{Materials and methods}

The study included subjects who had attended the general health check up program at Executive Health Scheme (EHS) department, Bombay hospital, Indore, Madhya Pradesh, India, where, patient's physical and medical history was taken and maintained in medical records department. These data were screened manually and total 726 individuals those having no medical history of diabetes, CVD and any other metabolic syndrome were chosen randomly.

Overnight fasted blood samples were collected in plain vacutainer tubes. The values of postprandial blood sugar (PPBS), TC, HDL and triglycerides (TG) were estimated directly by automated analyser (Xpand analyser). While, LDL, TC/HDL and LDL/HDL ratios were calculated out using standard formulas [9]. Prior to analysis, the analyser was calibrated with calibrators provided by the manufacturer. Controls were run at both normal and pathological concentrations for each analyte. During the course of the study there was no change in the equipment, reagents, calibration standards and controls. 


\subsection{Statistical analysis}

The analysis was performed using Prism software, version 5.1 for windows, Inc., La Jolla, CA, USA. The $\mathrm{P}<0.05$ was considered as statistically significant. A simple correlation analysis (Pearson's test) was utilized to calculate the correlations of PPBS with other studied factors.

\section{Results and Discussion}

In studied population, PPBS was found to be positively correlated with TG and TC/HDL ( $<<0.001$, for both), whereas a significantly negative correlation was observed between PPBS and HDL ( $\mathrm{P}<0.001)$. In case of other lipid profile parameters PPBS showed non-significant relation of PPBS with TC and LDL. Increased level of LDL and decreased HDL results in a high LDL/HDL ratio which was also found to be positively correlated with PPBS $(\mathrm{P}<0.001)$.

Fig.1: Correlation analysis of PPBS and HDL in sample population

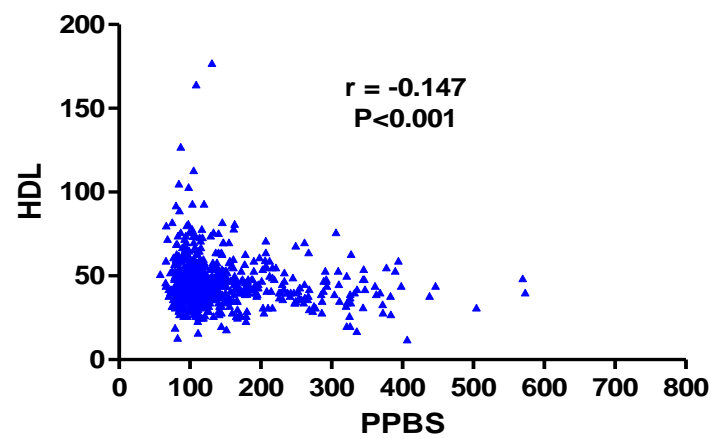

Where, PPBS, postprandial blood sugar; HDL, high density lipoprotein; r, correlation coefficient

Fig.2: Correlation analysis of PPBS and TG in sample population

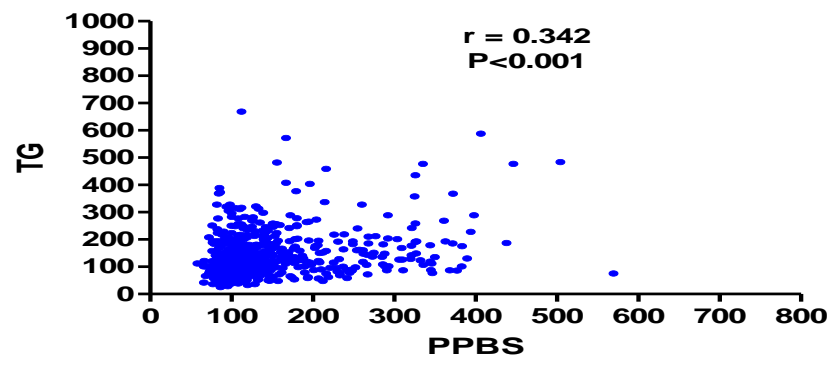

Where, PPBS, postprandial blood sugar; TG, triglyceride; $r$, correlation coefficient

The present observations revealed that the PPBS showed a direct association with TG and indirect association with HDL. Non-significant correlation was seen of PPBS with TC and LDL, might indicate a possible initiation of metabolic syndromes in the given population. Since, no prior study has been documented in this central Indian population; these results can be compared with other studies conducted in different regions of India. Studies from different regions of India including Delhi, Jaipur, Channai, Kochi $[6,10]$ Kerala, Mumbai, Kolkata, Hyderabad, Guwahati etc. also indicated a increased risk of metabolic syndrome in common people [11,12].

Fig.3 : Correlation analysis of PPBS and LDL in sample population

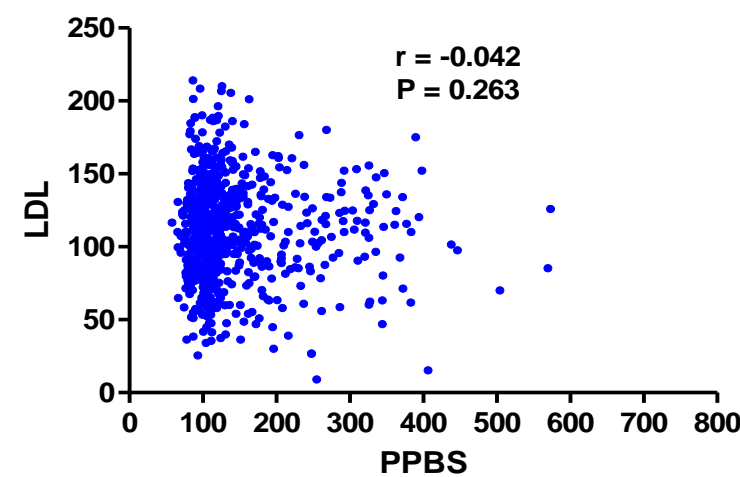

Where, PPBS, postprandial blood sugar; LDL, low density lipoprotein; r, correlation coefficient 
Assessment of association of postprandial blood sugar with lipid parameters in non-diabetic...

Fig.4: Correlation analysis of PPBS and TC in sample population.

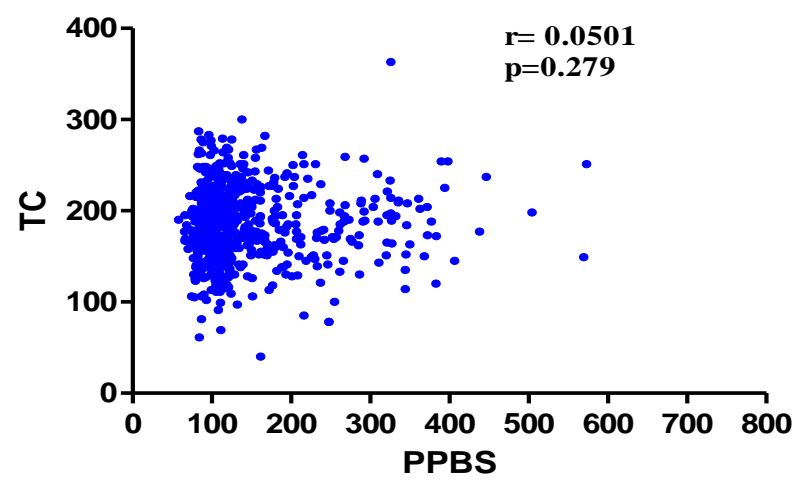

Where, PPBS, postprandial blood sugar; TC, total cholesterol; $r$, correlation coefficient

Fig.5: Correlation analysis of PPBS and TC/HDL in sample population.

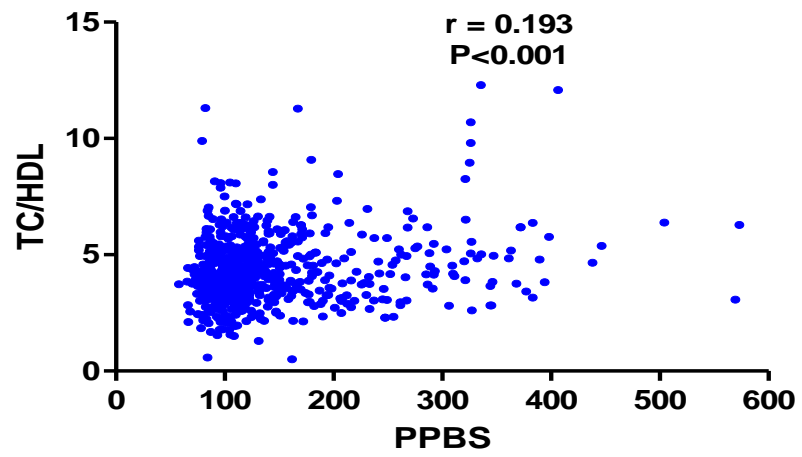

Where, PPBS, postprandial blood sugar; TC/HDL, Total cholesterol/high density lipoprotein; $r$, correlation coefficient

Fig.6 : Correlation analysis of PPBS and LDL/HDL in sample population

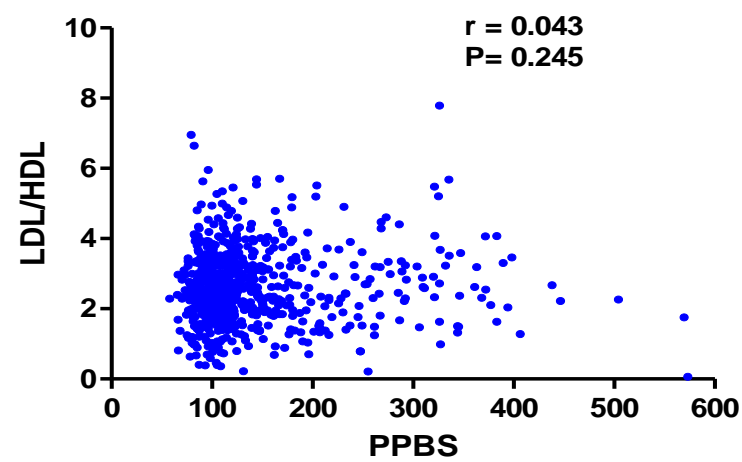

Where, PPBS, postprandial blood sugar; LDL/HDL, low density lipoprotein/ high density lipoprotein; r, correlation coefficient

Since, both carbohydrate and lipid metabolism are interconnected physiological pathways, obviously alteration in one affects the other. Clinical study, on tribal population of Andhra Pradesh, showed a strong link between serum sugar and lipid levels in diabetic patients [13-15]. Although with reference to non-diabetic healthy individual no such correlations were observed earlier [8] but, our test population demonstrated a significant correlation of PPBS with some of the studied parameters, probably this pointing out their prediabetic and unmanaged metabolic condition. In addition to this, Indian's genetic structure also makes them more susceptible for diabetes and associated CVD. Moreover, dietary factors, physical inactiveness play additional role. Eating of surplus fat diet may result in increased fat depot in liver, muscles and visceral organs that may enhance the rate of lipogenesis and leads to obesity. Gotto [17] has also found that increased serum level TG can induce CVD in normal as well as in diabetic patients $[7,13]$. 
Assessment of association of postprandial blood sugar with lipid parameters in non-diabetic...

Furthermore, the increased level of TC/HDL again indicated an increased possibility of CVD and ischemic heart disease in the studied population $[18,19]$. The concomitant increase in the values of PPBS and TG with affiliated decreased level of HDL can be compared with the findings of Durgawala et al [20] and Sanyal et al [21]. In some other cases such abnormal metabolic factors were also reported to be positively correlated with increased rates of non-alcoholic fatty liver disease (NAFLD) in obese patients [22]. Alternatively, enhanced value of TC/HDL and indicated an increased possibility of CVD and ischemic heart disease in the given population [12, 23].

Growing incidences of dyslipidemia with parallel increase in hyperglycaemia may be determine by the progress in the increasing consumption of sweetened beverages and high fat diet that may result in over production or lack of clearance of these lipoprotein particles, abnormally high serum lipid level [2, 24, 25]. Furthermore, the increased serum levels of TG have been found to be associated with increased plasma TNF $\alpha$ that may lead to insulin resistance by enhancing the production of inflammatory cytokines [26, 27]. In addition, elevated serum TG are also found to induce pancreatitis $[28,29]$ resulting in diabetes.

Surely, there is a need of bringing about dietary changes and identifying individuals that are at high risk of these disorders and then taking intensive intervention efforts $[2,11,23,30]$. These attempts can serve as effective ways to reduce/prevent the incidence of metabolic abnormalities and the ever increasing burden of diabetes and CVD in general population. In the present study, normal individuals showed a significant relation of PPBS with lipid parameters, indicated first need to identify these border lined prediabetic individuals that also have a higher risk of CVD and then to take intensive intervention efforts with regular health check up [19, 22, 31]. Furthermore, strong need for awareness programs to bringing about dietary changes, promote them for regular exercise and increased physical activity that may serve as effective ways to reduce/prevent the incidence altered metabolism and the constantly increasing burden of diabetes and CVD in general population.

\section{Conclusion}

In conclusion, a fairly positive correlation of PPBS with different cardiac risk factors was observed. Although, PPBS exhibited no direct correlation with TC and LDL and LDL/HDL, but a negative association was observed between PPBS and HDL, which may indicate an increased risk for CVD, not due increase in TC but due to abnormal TG and HDL level. Although, this study was conducted involving limited number of human samples, this appears to be the first report demonstrating statistically significant alterations in public health status. Finally, there is a need for large scale metabolic screening and it is recommended that the people should be made aware for their health status and to improve their routine life.

\section{Acknowledgement}

Financial support from University Grant Commission, New Delhi, India to Jyoti Agrawal (NET - JRF, Ref. no. 2120930513/20-12-2009 EU IV) and staff members of pathology dept., Bombay hospital, Indore for data collection, are gratefully acknowledged.

\section{References}

[1] Sawant A, Mankeshwar R, Shah S, Raghavan R, Dhongde G, Raje H, et al. Prevalence of metabolic Syndrome in Urban India. Cholesterol 2011; 2011:1-7.

[2] Pandit K, Goswami S, Ghosh S, Mukhopadhyay P, Chowdhury S. Metabolic syndrome in South Asians. Ind J Endocrinol Metab 2012; 16:44-55.

[3] Mohan V, Deepa M, Deepa R, Shanthirani CS, Farooq S. Secular trends in the prevalence of diabetes and impaired glucose tolerance in urban south India - The Chennai urban rural epidemiology study. Diabetalogia 2006; 49:1175-78.

[4] Agarwal N, Joshi S, Deshpande VK, Biswas DA. Correlation between glycated haemoglobin and glucose testing for diabetes mellitus screening. Indian J Med Sci 2013;67:149-54.

[5] Durgawale P, Patil S, Shukla PS, Sontakke A, Kakade S, Yadav S. Evaluation of reference intervals of serum lipid profile from healthy population in western Maharashtra. Ind j Clin Biochem 2009; 24:30-5.

[6] Gupta R, Sharma KK, Gupta A, Agrawal A, Mohan I, Gupta VP, et al. Persistent high prevalence of cardiovascular risk factors in the urban middle class in India: Jaipur Heart Watch-5. Indian Heart J 2012; 60:11-6.

[7] Haffner SM. Insulin resistance, inflammation, and the prediabetic state. Am J Cardiol 2003; 18:18-26.

[8] Sattar N. Revisiting the links between glycaemia, diabetes and cardiovascular disease. Diabetologia. 2013;56:686-95.

[9] Parmar HS, Kar A. Protective role of Citrus sinensis, Musa paradisiaca, and Punica granatum peels against diet-induced atherosclerosis and thyroid dysfunctions in rats. Nutr Res 2007; 27:710-18.

[10] Mohan, Pradeepa R. Epidemiology of diabetes in different regions of india. Health Administrator 2009; 22:1- 18

[11] Ashavaid TF, Todur SP, Dherai AJ. Health Status of Indian Population - Current Scenario. J Assoc Physicians of India 2004; 52 :363-69.

[12] Das SK, Faruque ASG, Chisti MJ, Ahmed S, Mamun AA, Chowdhury AK, et al. Nutrition and Lipid Profile in General Population and Vegetarian Individuals Living in Rural Bangladesh. J Obes Wt Loss Ther 2012; 2:1-5.

[13] Samatha P, Venkateswarlu M, Siva Prabodh V. Lipid Profile Levels in Type 2 Diabetes Mellitus from the Tribal Population of Adilabad in Andhra Pradesh, India. Clin Diagnos Res 2012; 6:590-2.

[14] Idogun ES, Unuigbe EI, Ogunro PS, Akinola OT, Famodu AA. Assess $\neg$ ment of the serum lipids in Nigerians with type 2 diabetes mellitus complications. Pak. J. Med. Sci. (Part 1) 2007; 2:708-12.

[15] Albrki WM, Elzouki AN Y, EL-Mansoury ZM, Tashani OA. Lipid profiles in Libian type 2 diabetes. J Sci Appls 2007; 1:18-23.

[16] Sattar N. Revisiting the links between glycaemia, diabetes and cardiovascular disease. Diabetologia. 2013;56:686-95.

[17] Gotto AM. Triglyceride as a risk factor for coronary artery disease. Jr Am J Cardiol 1998;82:22-5. 
Assessment of association of postprandial blood sugar with lipid parameters in non-diabetic...

[18] Lorenzo C, Hartnett S, Hanley AJ, Rewers MJ, Wagenknecht LE, Karter AJ, Haffner SM. . Impaired fasting glucose and impaired glucose tolerance have distinct lipoprotein and apolipoprotein changes: the insulin resistance atherosclerosis study. J Clin Endocrinol Metab 2013; 98:1622-30.

[19] Penno G, Solini A, Bonora E, Fondelli C, Orsi E, Zerbini G, Trevisan R, Vedovato M, Gruden G, Laviola L,Nicolucci A, Pugliese G. Gender differences in cardiovascular disease risk factors, treatments and complications in patients with type 2 diabetes: the RIACE Italian multicentre study. J Intern Med. 2013; 274:176-91.

[20] Durgawale P, Patil S, Shukla PS, Sontakke A, Kakade S, Yadav S. Evaluation of reference intervals of serum lipid profile from healthy population in western Maharashtra. Ind j Clin Biochem 2009; 24:30-5.

[21] Sanyal D, Ghosh S, Mukherjee P, Mukherjee S, Chowdhury S. Dyslipidemia, metabolic syndrome, and liver enzymes in impaired glucose tolerance and new onset untreated, type 2 diabetes Indian subjects. Indian J Endocrinol Metab 2012; 16:434-5.

[22] Saran S, Philip R, Gutch M, Tyagi R, Agroiya P, Gupta KK. Correlation between liver fat content with dyslipidemia and Insulin resistance. Indian J Endocrinol Metab 2013; 17:355-7.

[23] Dobiasova M, Frohlich J. The plasma parameter $\log$ (TG/HDL-C) as an atherogenic index: correlation with lipoprotein particle size and esterification rate in apob-lipoprotein-depleted plasma (FERHDL). Clin Biochem 2001; 34:583-8.

[24] Das M, Saikia M. Estimation of reference interval of lipid profile in Assamese population. Ind J Clin Biochem, 2009; 24:190-93.

[25] Malik VS, Popkin BM, Bray GA, Despres JP, Willett WC, Hu FB. Sugar-sweetened beverages and risk of metabolic syndrome and type 2 diabetes: a meta-analysis. Diabetes Care 2010; 33:2477-83.

[26] Edwardson CL, Gorely T, Davies MJ, Gray LJ, Khunti K, Wilmot EG, et al. Association of sedentary behaviour with metabolic syndrome: a meta-analysis. Plos One 2012; 7:34916.

[27] Tiwari S, Sadashiv, Paul BN, Kumar S, Chandra A, Dhananjai S, Negi MP. TNF- $\alpha$ Gene Expression in Subcutaneous Adipose Tissue Associated with HOMA in Asian Indian Postmenopausal Women. Horm Metab Res 2013 [in press].

[28] Klop B, Elte JWF, Cabezas MC. Dyslipidemia in Obesity: Mechanisms and Potential Targets. Nutrients 2013; 5 : 1218-40;

[29] Chopra SM, Misra A, Gulati S, Gupta R. Overweight, obesity and related non-communicable diseases in Asian Indian girls and women. Eur J Clin Nutr 2013; 67:688-96.

[30] Misra A, Shrivastava U. Obesity and dyslipidemia in South Asians. Nutrients 2013; 15:2708-33.

[31] Sadikot SM, Singh V. Managing diabetes in India: paradigms in care--outcomes and analysis in a comprehensive, clinical practice survey of Indian physicians. J Indian Med Assoc 2011; 109:844-8. 